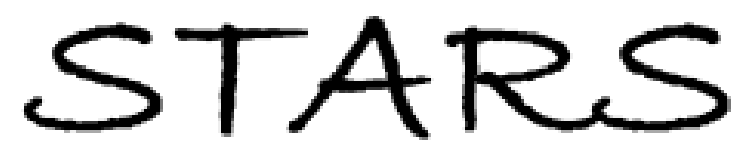

University of Central Florida

STARS

$1-1-2000$

\title{
Evidence of scattering anisotropy effects on boundary conditions of the diffusion equation
}

\author{
G. Popescu \\ University of Central Florida \\ C. Mujat \\ University of Central Florida
}

A. Dogariu

University of Central Florida

Find similar works at: https://stars.library.ucf.edu/facultybib2000

University of Central Florida Libraries http://library.ucf.edu

This Article is brought to you for free and open access by the Faculty Bibliography at STARS. It has been accepted for inclusion in Faculty Bibliography 2000 s by an authorized administrator of STARS. For more information, please contactSTARS@ucf.edu.

\section{Recommended Citation}

Popescu, G.; Mujat, C.; and Dogariu, A., "Evidence of scattering anisotropy effects on boundary conditions of the diffusion equation" (2000). Faculty Bibliography 2000s. 2747.

https://stars.library.ucf.edu/facultybib2000/2747

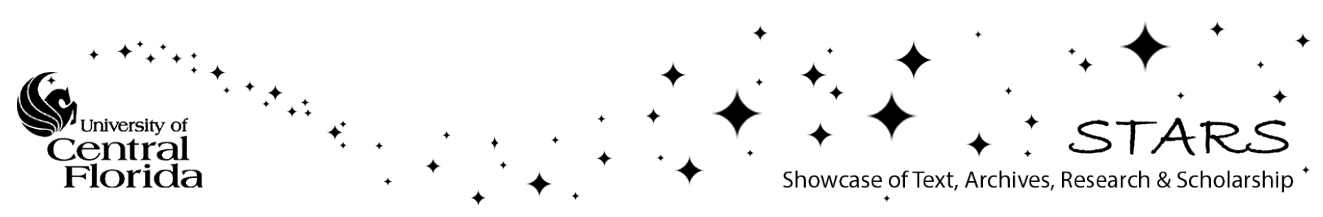




\title{
Evidence of scattering anisotropy effects on boundary conditions of the diffusion equation
}

\author{
G. Popescu, C. Mujat, and A. Dogariu \\ School of Optics/CREOL, University of Central Florida, Orlando, Florida 32816 \\ (Received 30 July 1999; revised manuscript received 8 November 1999)
}

\begin{abstract}
We present experimental evidence that the diffusion process close to the boundary is fundamentally different from the bulk diffusion in the sense that it is affected by the scattering properties of the individual centers. Systematic measurements show a nontrivial behavior of the extrapolation length ratio on the scattering anisotropy of individual particles. The results are compared with other works and are supported by an independent Monte Carlo simulation. A simple analytical model that emphasizes the influence of the last scattering event successfully describes the results.
\end{abstract}

PACS number(s): 42.68.Ay, 42.25.Gy, 42.25.Kb

\section{INTRODUCTION}

Recently, remarkable advances in fundamental understanding and experimental methodologies proved that light propagation in random media is a source of unexplored physics with a wide range of potential applications [1]. Among them, the medical applications occupy a special place, since it has been proven that scattering of optical radiation can be successfully used as a noninvasive investigation technique $[2,3]$.

The wave transport in a medium consisting of isotropic scattering centers is characterized by two different length scales: the scattering mean free path, which is the average distance between the scattering events, and the absorption length, which characterizes the light attenuation due to absorption inside the medium. When the medium is highly scattering, the wave transport is essentially diffusive. Under these circumstances the wave propagation can be described by the diffusion equation, which brings along important simplifications to the wave transport treatment [4].

For infinite media and different source geometries, solutions to the diffusion equation can be easily obtained, usually in closed forms. However, when the scattering medium is bounded, special conditions are needed to set the behavior of energy density at the interfaces. Through the phenomenon of reflection at the interface, the light is reinjected into the medium and forced to travel a new diffusive path inside the scattering medium. As the reflectivity increases, this process becomes gradually important and it has to be taken into account in the overall diffusive description of the light propagation in the bounded scattering medium. The diffusion process at the boundary introduces a new scale length that characterizes the specific medium interface. This is the "extrapolation length," which measures the distance outside the medium where the energy density vanishes linearly. Accurate value of this length is needed to interpret any experiment based on diffusion of light.

In general, it is well understood that the extrapolation length depends on the refractive index mismatch at the boundary but, so far, little has been done to clarify the potential influence of scattering anisotropy on boundary phenomena. For quite some time it was commonly accepted that the main effect of anisotropic scattering is to rescale the diffusion lengths in the bulk and this procedure has been straightforwardly expanded to treat the boundary conditions. Scattering anisotropy effects were signaled in the angular dependence of light backscattering from diffuse systems [5]. This result was interpreted as being due to a discontinuity of the photon concentration at the source point. In another attempt, the influence of the scattering anisotropy on the extrapolation length ratio has been theoretically studied solving the exact radiative transfer problem [6,7]. Unfortunately, the complexity of calculations allows analytical solutions only for extreme values of anisotropy factors and reflectivities at the boundaries. In a recent paper, Chen and Bai present a unique numerical approach and show that, in fact, more careful analysis is needed for the subtle effects at the interface between diffusive and nondiffusive media [8].

Our goal in the present paper is to comprehensively study the effect of both reflection at the boundary and single scattering anisotropy on the extrapolated length. The experimental method [9] is based on the principle of low coherence interferometry and it is applied here for the first time in investigations of diffusion at the boundary. The experimental results are compared with numerical simulations and other literature data and are also supported by an analytical description which emphasizes the effects of the last scattering event on the overall angular distribution of the backscattered waves.

\section{BOUNDARY CONDITION FOR SEMI-INFINITE MEDIA}

In many situations of practical interest, the light propagation through dense scattering media can be assumed to be diffusive and consequently described by a diffusion equation. This is the case when, within the random medium, the waves travel over distances much larger than the transport mean free path $\left(l_{t}\right)$ and the absorption is not considerable. Mathematically, the angular flux is considered anisotropic only in the first order [4],

$$
\varphi(\mathbf{r}, \boldsymbol{\Omega}, t) \simeq \frac{1}{4 \pi} \Phi(\mathbf{r}, t)+\frac{3}{4 \pi} \mathbf{j}(\mathbf{r}, t) \mathbf{\Omega},
$$

where $\boldsymbol{\Omega}$ is the direction unit vector, while $\Phi$ and $\mathbf{j}$ are the energy density and flux, respectively. Using Fick's formula, 
$\mathbf{j}(\mathbf{r}, t)=-D \nabla \Phi(\mathbf{r}, t)$, the diffusion equation can be obtained in terms of $\Phi$ alone and, for media of negligible absorption, it takes the form

$$
\left(\frac{\partial}{\partial t}-D \nabla^{2}\right) \Phi(\mathbf{r}, t)=S(\mathbf{r}, t) .
$$

In the last equation, $D$ is the diffusion coefficient of the medium, while $S$ is the source term, considered to be isotropic. In order to solve the diffusion equation for finite media, one needs to specify boundary conditions and several alternatives have been proposed [10]. The problem of semiinfinite random media becomes more complex as the reflection at the boundary has to be taken into account. This is not possible using the separate Dirichlet-Neuman boundary conditions because setting the energy density to be zero at the interface is incorrect and does not correspond to the physical situation. When the refractive index of the diffusive medium is higher than that of the outer medium, the photons trying to exit the sample are resent back mostly by the total internal reflection process. Lagendijk, Vreeker, and DeVries [11] solved the diffusion equation for different geometries using modified Green's functions to take into account the reflection at the boundary. Their results showed that the effect of reflection lowers the effective diffusion coefficient of the medium. An appropriate boundary condition is, therefore, desirable and may extend the applicability of the diffusion model close to the interface.

The most general approach is to use a mixed boundary condition, which for a semi-infinite medium can be written as

$$
\left[\Phi-z_{e} l_{t} \frac{\partial \Phi}{\partial z}\right]_{z=0}=0
$$

where $z_{e}$ is called the extrapolated length ratio, since $z_{e} l_{t}$ is the distance outside the medium where $\Phi$ extrapolates to zero. This relation uniquely defines the behavior of the diffusion process at the plane $z=0$. Freund [12] has derived the boundary conditions for one, two, and three dimensions from both the transport theory and Milne equations and found that, for the one-dimensional case, they are consistent with each other, while for the two- and three-dimensional cases, discrepancies appear for the values of the extrapolation length. Using a partial current technique, Zhu, Pine, and Weitz showed that $z_{e}$ in Eq. (3) depends only on the reflection phenomenon at the boundaries and is given by [13]

$$
z_{e}=\frac{2}{3} \frac{1+R_{\mathrm{eff}}}{1-R_{\mathrm{eff}}}
$$

In Eq. (4) $R_{\text {eff }}$ is the effective reflectivity at the interface and can be easily calculated using the first two moments of the Fresnel coefficient, averaged for the two polarizations. For a boundary without reflections, or, alternatively, for a totally absorbing interface, $z_{e}$ takes the value $2 / 3$, which is consistent with the diffusion theory [1]. However, as $R_{\text {eff }}$ increases, in situations where the total internal reflection is present, the value of the extrapolation length ratio predicted by Eq. (4) can be 2-3 times larger [14].
As can be seen from Eq. (3), the parameter $z_{e}$ is of the utmost importance since it solely defines the boundary condition for a given system. Therefore, an experimental way to determine the extrapolation length for a given random medium is highly desirable. So far, the angular diffuse transmission has been the only technique used to measure $z_{e}$; the results agree with those given by Eq. (4) and show no dependence on the scattering anisotropy [14]. However, recently the Monte Carlo method introduced by Chen and Bai predicted a rather significant effect of the scattering anisotropy on the extrapolation length ratio [8]. In the following, we will investigate the diffusion at the boundary in order to quantify both reflectivity and anisotropy effects.

\section{EXPERIMENTAL RESULTS}

Recently, optical path-length spectroscopy (OPS) has been implemented as a technique for investigating the wave propagation through multiple scattering media [9]. We apply this method to measure the values of $z_{e}$ for random media with various anisotropy factors $g$ and with different reflecting boundaries. In a Michelson interferometer with a low coherence source, OPS combines the field coming from a reference mirror with the one backscattered from the diffusive medium. By tuning the optical length of the reference arm and detecting the interference term, one obtains the optical path-length distribution of light backscattered from the sample. The detected intensity has the form $I_{d}=I_{s}+I_{\text {ref }}$ $+2 \sqrt{I_{s} I_{\text {ref }}}|\gamma(\Delta s)| \cos (2 \pi \Delta s / \lambda+\varphi)$, where $I_{d}, I_{s}$, and $I_{\text {ref }}$ are the detected, scattered, and reference intensities, respectively, whereas $\varphi$ is the phase associated with the complex degree of coherence $\gamma(\Delta s)$. The signal is characterized by a high dynamic range due to the heterodyne detection and a high resolution in the optical path-length domain ensured by the short coherence length of the source. In our experiments, the light source is a light-emitting diode with a wavelength $\lambda=1.3 \mu \mathrm{m}$ and the coherence length of $10 \mu \mathrm{m}$. The detection is polarization independent as described in Ref. [9] and the same single-mode fiber is used to send and collect the light from the random medium. In this configuration, the incident beam is narrow and normal to the free surface of the scattering medium. Throughout our experiments, the dimensions of the samples are much larger than the corresponding transport mean free paths, therefore, a semi-infinite geometry is considered. In the frame of the diffusion equation, the path-length resolved backscattered flux is obtained solving Eq. (2) together with the boundary condition given in Eq. (3) and, for negligible absorption, it has the form

$$
J(s)=A l_{t}^{-3 / 2} z_{e} s^{-5 / 2} \exp \left(-\frac{3 z_{e}^{2} l_{t}}{4 s}\right) .
$$

Here $s$ is the distance traveled through the medium and $A$ is a constant which depends on the source strength.

The samples used in these experiments are polystyrene microspheres of three different dimensions suspended in water. The scattering function anisotropies are characterized in terms of $g$, the average cosine of the scattering angle, and have the values of $0.32,0.49$, and 0.93 . The corresponding values of the transport mean free paths are equal to 55, 40, and $180 \mu \mathrm{m}$, as derived from Mie theory. When compared to 

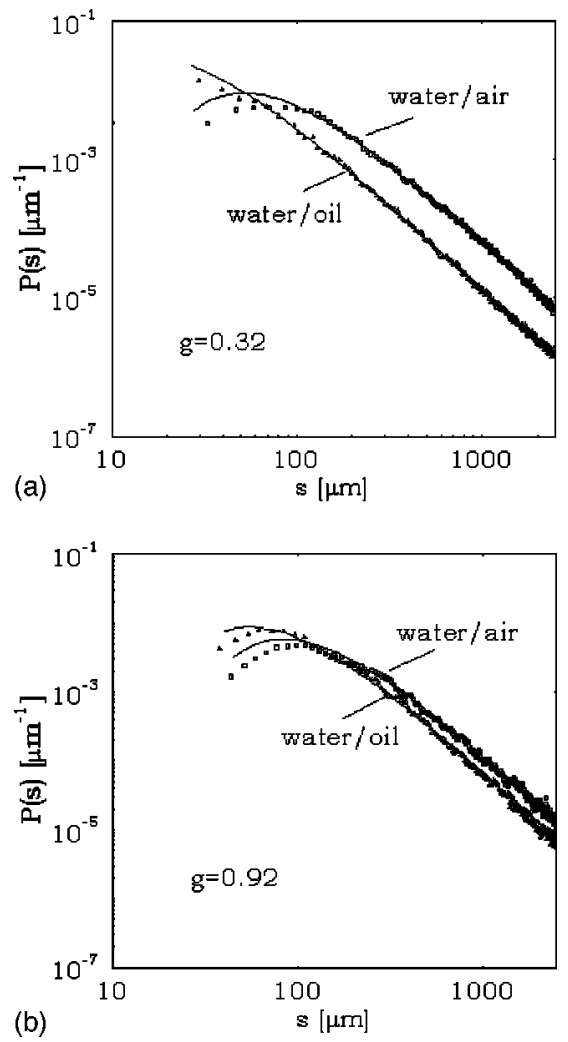

FIG. 1. Probability density of backscattered light for media $g$ $=0.32$ (a) and $g=0.92$ (b) and for two different interfaces, as indicated. The solid curves represent the fit with the diffusion model of Eq. (5) normalized.

scattering, the absorption is negligible in these samples (absorption coefficient is of the order of $1 \mathrm{~cm}^{-1}$ ). For each sample, two successive measurements were performed. The OPS backscattered signal was first recorded at the water/air interface of the sample. In this case, the condition for total internal reflection is present, since the backscattered light encounters at the boundary of medium of lower refractive index. In order to change the boundary conditions, a layer of optical oil was added on top of the scattering medium. Because of its lower density, the oil and water do not mix. In addition, due to the high viscosity of the oil, the polystyrene particles are prevented from migrating into the layer of oil. Thus, we created a new sharp interface, with different refractive properties. The thickness of the oil layer is sufficiently large and the optical fiber is immersed close to the surface of the medium. The refractive index of the oil is measured to be $n=1.39$.

After detection, the path-length resolved signal was normalized with the area under the curve to obtain the probability density of backscattered waves. Typical experimental results are presented in Figs. 1(a) and 1(b), where $P(s)$ represents the probability that the backscattered light has traveled in the medium an optical path-length within the interval $[s, s+d s]$. To our knowledge, this is the first time that direct measurements of $P(s)$ are accessible and, moreover, that direct experimental evidence of boundary effects on $P(s)$ is shown. It can be easily observed that the reflection at the boundary changes dramatically the optical path-length distribution of waves in the medium in a sense that the probability of long optical path lengths is increased for a highly reflective boundary. Consequently, the average penetration depth of light in the medium is considerably increased. The continuous curves in Fig. 1 are the result of data fitting with the normalized version of the diffusion model given by Eq. (5). Because $l_{t}$ and $g$ are known, the only fitting parameter used is the extrapolation length ratio $z_{e}$. The values of $z_{e}$ calculated with Eq. (4) are 0.68 for the medium/oil interface and 1.67 for the medium/air interface. Therefore, the oil interface simulates an almost perfect absorbing plane at the boundary of the scattering medium, for which $z_{e}=0.67$. The experimental values obtained for the medium/oil boundary are $0.74,0.73$, and 0.76 , corresponding to the anisotropy factors of $0.32,0.49$, and 0.92 , respectively. These values are slightly different from that of 0.67 predicted by Eq. (4) and closer to the value of 0.7104 given by the Milne problem for isotropic scattering. As expected, for this index-matched interface, the anisotropy does not have a significant effect on $z_{e}$. This result is in agreement with the theoretical findings of Refs. [6,7], which, for no reflection at the boundary, predict values of $z_{e}$ within the interval from 0.7092 to 0.7150 for different anisotropies. However, for the medium/air interface, the experimental values are $1.57,1.43$, and 1.12, respectively, which are significantly different from that of 1.67 calculated with Eq. (4). Thus, the measured trend for $z_{e}$ is to decrease as $g$ increases; experimentally we obtained a minimum value of 1.12 corresponding to $g=0.92$. This value is considerably smaller than the calculated one and it suggests that the anisotropy dependence of the extrapolated length ratio becomes stronger at higher values of $g$.

Our results for the medium/air boundary do not agree with the simulations and experiments presented in Refs. [1416] which have come to the common conclusion that the anisotropy factor does not play any role on the angularresolved diffuse transmission. Vera et al. [5] present simulations and experimental results for angular-resolved light backscattering from diffusive media. It is shown that the angular distribution of backscattered light depends not only on the reflection at the boundary, but also on the anisotropy factor of each individual scattering event. Following the approach used initially in the one-dimensional case, this result is modeled within the diffusion theory by a discontinuity in the photon concentration at the source point, which is proportional with the anisotropy factor. The anisotropy factor $g$ is included in the expression of an exponentially decaying source and, therefore, the angular backscattering distribution is affected by $g$ mainly in the low-order scattering components. However, the extrapolation length ratio used here $z_{e}$, is $g$ independent and is calculated using the procedure introduced by Zhu, Pine, and Weitz (ZPW) [13]. One should note that due to the intrinsic nature of reflectance measurements, the low-order scattering contributions dominate and, therefore, determine the overall angular distribution of backscattered light. Upon diffusion through long propagation paths, the effect of an anisotropic source is lost, as Vera, Lemieux, and Durian also mention in their paper [5]. Accordingly, angular-resolved transmission, which probes only higherorder scattering paths, cannot reveal information regarding the source anisotropy. Our measurements and simulations, on the other hand, offer the path-length resolved distribution of the backscattered light and deal with all scattering orders. 
The results show that the anisotropy factor has an important influence on the extrapolation length ratio, $z_{e}$.

It is worth mentioning that angular-resolved measurements are usually performed in air and the light scattered at angles larger than the critical angle does not exit the sample and cannot be detected. Therefore, the angular-resolved experiments offer access only to a partial angular distribution of scattered light, which may not be sufficient to observe the anisotropy effects on the extrapolation length ratio. As opposed to such measurements, the high dynamic range OPS signal incorporates the global effect of reflection at the boundary, including the internally reflected component. Due to the versatility associated with this method, the measurements are performed at the open surface of the medium and the reflectivity can be evaluated accurately, without complications caused by double and multiple reflections introduced by a second dielectric interface. As a result, the experiment presented here is able to demonstrate not only the expected importance of the reflection at the boundary, but also that, for a given interface, the anisotropy factor changes the diffusion mechanism at the interface.

Anisotropy-dependent behavior of the extrapolated length ratio has also been observed in the numerical simulations in Refs. [17,18], but, unfortunately, there only the situation of the totally absorbing interface is investigated. As mentioned before, Chen and Bai have proposed a numerical technique to obtain the boundary condition for the diffusion equation (8). Their work includes the case of boundary mismatch and the results clearly show that $z_{e}$ becomes consistently lower for higher values of $g$. However, no explanation for this result is given. For a situation equivalent to our medium/air boundary, they report a value $z_{e} \simeq 1.0$ corresponding to $g$ $=0.9$, which is compatible to our value $z_{e}=1.12$ obtained for $g=0.92$. The experimental results presented in this paper are evidence of the scattering anisotropy effect on the extrapolation length ratio in the context of diffusion equation. In the following sections, we provide results of a numerical experiment, as well as a simple model in support of these observations.

\section{NUMERICAL EXPERIMENT-MONTE CARLO SIMULATION}

To check the experimental findings presented in the preceding section and to get more insight into possible $z_{e}(g)$ effects, we have also performed a comprehensive numerical experiment: a Monte Carlo simulation of photon transport inside a semi-infinite medium. The optical properties of the medium $-l_{a}$, mean absorption length; $l_{s}$, mean free path; $g ; n_{i}$, index of refraction inside the medium; $n_{o}$, index of refraction outside the medium - are all variable. Multiple data files containing the properties of the photons returning into the semispace $z<0$ are recorded and then used to get the path-length-resolved reflectivity. Subsequently, $P(s)$ is fitted with Eq. (5) to get the extrapolation ratio $z_{e}$ for a given $l_{t}$ and anisotropy parameter $g$.

In the numerical experiment, photons are launched one by one into the medium in a pencil beam configuration, normally incident on the slab, to recover the experimental geometry. For each photon, an absorption length is randomly selected from an exponentially decaying distribution charac- terized by $l_{a}$. The photon trajectory is then simulated, both scattering and reflections at the boundaries being taken into account. The process starts from the origin of the coordinate system and the first scattering event takes place along the $O z$ axis. For all other scattering events, three random numbers are generated to determine the distance to the next scattering point and the polar and azimuthal angle that define the photon propagation direction after the scattering. The distance between two scatterings is sampled from an exponentially decaying distribution characterized by $l_{s}$. The azimuthal angle $\phi$ is chosen to be any angle between 0 and $2 \pi$, while the polar angle $\theta$ is sampled from the Henyey-Greenstein distribution [19] because it has a simple analytical form that can be used to express both isotropic and forward peak distributions by modifying the anisotropy factor $g$.

Reflection at the boundary is included as a function of the incidence angle $\theta$ by means of Fresnel equations averaged over both polarization states. We keep track of the photon path length and if it becomes larger than the predetermined absorption length, we discard it. For this particular experiment, $l_{a}$ is chosen to be 100 times larger than the transport mean free path $\left(l_{t}\right)$ such that the absorption can be considered negligible. Whenever the value of $z$ becomes negative, we record the set of coordinates $(x, y, z)$, direction $(\theta, \phi)$, number of scattering events, and optical path length regardless of the distance from the launch position at which that photon was reflected from the slab.

In general, a large number of detected photons is required to reduce the statistical variance. This means that the time necessary for collection over a small area becomes prohibitively long. However, similar information on $z_{e}$ can be obtained by integrating the reflected photons over the entire area of the sample and using the expression derived for the path-length-resolved integrated reflectance to fit our data [20]:

$$
R(s)=B l_{t}^{1 / 2} z_{e} s^{-3 / 2} \exp \left(-\frac{3 z_{e}^{2} l_{t}}{4 s}\right),
$$

where, $B$ is a constant that depends on the source strength and the light velocity inside the medium.

Simulations have been performed for $g=0.1,0.32,0.49$, and 0.75 and a decrease in the extrapolation length is obtained as the anisotropy factor $g$ increases (see data in Fig. $3)$. The results are consistent with previous Monte Carlo calculations [8] and are also in agreement with the experimental data presented in Sec. III.

\section{LAST-SCATTERING-DEPENDENT BOUNDARY CONDITIONS}

In general, the transport theory is extended to anisotropic scatterers by rescaling the mean free path that characterizes the medium to the transport mean free path $l_{t}=l_{s} /(1-g)$ [1]. Thus, the average distance over which the waves randomize their directions of propagation is increased. Formally, the medium is treated as a fictitious system of isotropic scatterers characterized by a larger average step between two scattering events. Zhu, Pine, and Weitz (ZPW) have found a relationship between the diffusion at the boundary and the reflectivity of the boundary, which has a particularly 


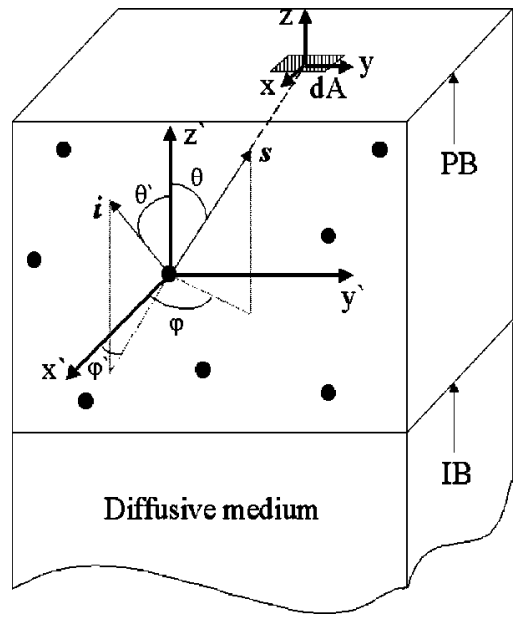

FIG. 2. Geometry of a last scattering event in a fictitious skin layer bounded by a physical boundary (PB) and an imaginary boundary (IB). The incident and the scattering directions are $\mathbf{i}$ and $\mathbf{s}$, respectively.

simple form [13]. As discussed earlier, for isotropic scattering, the reflectivity effects can be taken into account by modifying the boundary condition of the diffusion equation. It has been suggested that this treatment can be extended to anisotropic scattering simply by replacing $l_{s}$ with $l_{t}$ [13].

Undoubtedly, the results of our experiments and simulations presented in the previous sections show that the extrapolation length ratio depends on the anisotropy of individual scattering events. This conclusion is not predicted by the model implemented by ZPW and it becomes apparent that by simply rescaling the mean free path, one cannot account for such subtle boundary effects. In the following, we show that the boundary condition given in Eq. (3) can be modified to account for a dependence on the scattering anisotropy. The main idea is that, although the light transport is essentially diffusive in the bulk, the angular distribution of waves at the boundary is shaped by the last scattering event.

Let us consider a diffusive medium bounded by a plane perpendicular on $O z$ axis, as shown in Fig. 2. In addition, we separate a superficial layer by taking an imaginary boundary (IB) inside the medium such that the last scatterers are contained within the volume $V_{i}$ limited by the IB and physical boundary (PB). The waves passing IB are considered to be completely diffusive and, therefore, characterized by an angular transmission probability given by [14]

$$
T(\theta, \varphi)=\frac{1}{2 \pi} \frac{\gamma \cos (\theta)+\cos ^{2}(\theta)}{\gamma / 2+1 / 3},
$$

where $\gamma$ is the extrapolation length ratio with respect to IB. Because this virtual layer is index matched with the bulk, there is no reflection at the imaginary boundary and, consequently, $\gamma$ takes the value $2 / 3$. For each particle in this layer, the probability to scatter the light within the elementary solid angle $d \Omega=\sin \theta d \theta d \varphi$ is well approximated by the HenyeyGreenstein formula,

$$
h_{g}(\alpha)=\frac{1}{4 \pi} \frac{1-g^{2}}{\left(1+g^{2}-2 g \cos \alpha\right)^{3 / 2}},
$$

where $\alpha$ is the angle between the incident $\mathbf{i}$ and the scattering $\mathbf{s}$ direction. Thus, the angular distribution of light scattered by the particles contained in the volume $V_{i}$, when the incident light has the profile given by Eq. (7), can be expressed as

$$
F_{g}(\theta, \varphi)=C \int_{0}^{2 \pi} \int_{0}^{\pi / 2} h_{g}(\alpha) T\left(\theta^{\prime}, \varphi^{\prime}\right) \sin \left(\theta^{\prime}\right) d \theta^{\prime} d \varphi^{\prime},
$$

where $C$ is a constant such that $\int_{0}^{2 \pi} \int_{0}^{\pi / 2} F_{g}(\theta, \varphi) \sin (\theta) d \theta d \varphi$ $=1$. Using the notations in Fig. 2,

$$
\cos (\alpha)=\cos \left(\theta^{\prime}\right) \cos (\theta)+\sin \left(\theta^{\prime}\right) \sin (\theta) \cos \left(\varphi^{\prime}-\varphi\right) .
$$

In Eq. (9), $F_{g}(\theta, \varphi)$ represents the angular distribution of light emerging from one last scatterer which is exposed to the incident diffusive field. It can be shown that $F_{g}(\theta, \varphi)$ is independent of $\varphi$, consistent with the cylindrical symmetry of the problem. The next step is to find the diffusive energy flux scattered through the elementary area $d A$ included in the physical boundary. The major assumption we make at this point is that the scattering from a volume element $d V$ obeys the angular distribution given in Eq. (9). Thus, the flux scattered from the elementary volume $d V$ and passing through the area $d A$ is

$$
d J=c \Phi(r, \theta, \varphi) F(\theta, \varphi) \frac{d A \cos (\theta)}{r^{2}} d V e^{-r / l_{t}}
$$

where $c$ is the speed of light in the medium and $d V$ $=r^{2} \sin (\theta) d \theta d \varphi$. The total flux per unit area can be obtained by integrating over the entire volume. The inward integrated flux, corresponding to the $-z$ semispace has the form

$$
\begin{aligned}
j_{-}= & \frac{c}{4 \pi} \int_{-\pi / 2}^{0} d \theta \int_{0}^{2 \pi} d \varphi \int_{0}^{\infty} d r F_{g}(\theta, \varphi) \\
& \times \Phi(r, \theta, \varphi) \cos (\theta) \sin (\theta) e^{-r / l_{t}} .
\end{aligned}
$$

For a PB without reflection, the flux $j_{-}$must vanish. However, when an index mismatch is present, the light is resent into the medium through the process of reflection at PB. In this case, the inward component is equal to the outward one times the reflection coefficient of the interface

$$
\begin{aligned}
j_{-}= & \frac{c}{4 \pi} \int_{0}^{\pi / 2} d \theta \int_{0}^{2 \pi} d \varphi \int_{0}^{\infty} d r R(\theta) F_{g}(\theta, \varphi) \\
& \times \Phi(r, \theta, \varphi) \cos (\theta) \sin (\theta) e^{-r / l_{t}} .
\end{aligned}
$$

In the preceding equation, $R(\theta)$ is the Fresnel coefficient averaged for the two polarizations. Using the first order Taylor expansion of $\Phi(r, \theta, \varphi)$ about the origin [13], we can equalize the expressions (12) and (13). After some mathematical manipulations, the result can be arranged in the form

$$
\left.\Phi\right|_{z=0}-\left.l_{t} \frac{b+R_{j}}{a-R_{\phi}} \frac{\partial \Phi}{\partial z}\right|_{z=0}=0
$$

where the parameters are defined as follows: 


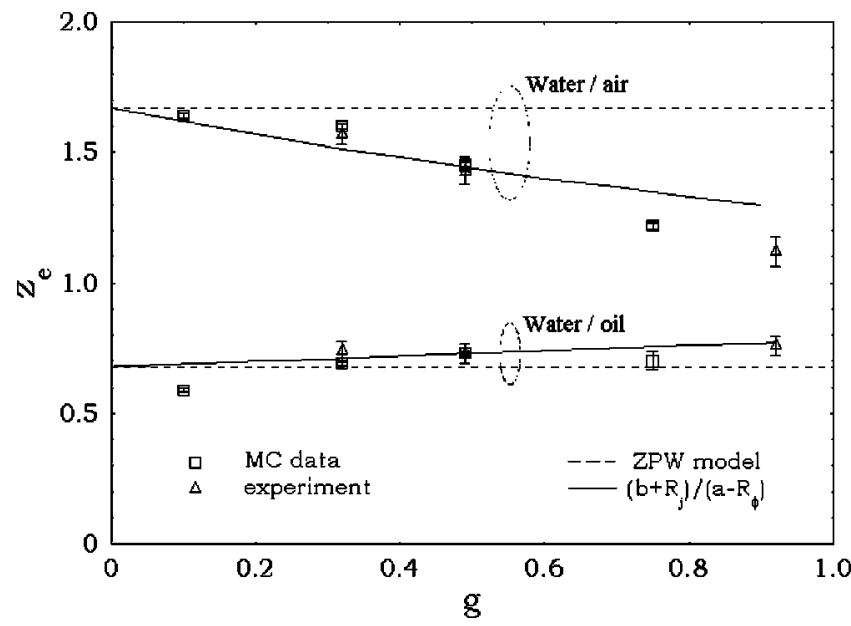

FIG. 3. Values for $z_{e}$ as obtained from the OPS experiment, numerical simulation, the ZPW model, and the present model. The error bars in the experimental and the simulation data are also shown. The data are separated in two groups corresponding to the two boundaries, as indicated.

$$
\begin{gathered}
a=\int_{0}^{2 \pi} \int_{0}^{\pi / 2} F_{g}(\theta, \varphi) \sin (\theta) \cos (\theta) d \theta d \varphi \\
b=\int_{0}^{2 \pi} \int_{0}^{\pi / 2} F_{g}(\theta, \varphi) \sin (\theta) \cos ^{2}(\theta) d \theta d \varphi \\
R_{\phi}=\int_{0}^{2 \pi} \int_{0}^{\pi / 2} F_{g}(\theta, \varphi) R(\theta) \sin (\theta) \cos (\theta) d \theta d \varphi \\
R_{j}=\int_{0}^{2 \pi} \int_{0}^{\pi / 2} F_{g}(\theta, \varphi) R(\theta) \sin (\theta) \cos ^{2}(\theta) d \theta d \varphi .
\end{gathered}
$$

The extrapolation length ratio that we introduce, $z_{e}=(b$ $\left.+R_{j}\right) /\left(a-R_{\phi}\right)$, depends on the anisotropy factor in a manner shown in Fig. 3. As expected, for the case of isotropic scattering, $F_{g}(\theta, \varphi)$ becomes a constant and the values for $z_{e}$ are identical with those obtained using the ZPW approach. In the situation where the reflection at the boundary is close to zero, $z_{e}$ has a slight dependence on $g$ in such a way that increases for higher anisotropy factors. The monotonic behavior of the function $z_{e}(g)$ for medium/oil interface is reversed with respect to the medium/air case because now the light exits the sample into a medium with a higher index of refraction. As can be seen in Fig. 3, the $z_{e}$ values predicted by this model are fairly close to the measured and simulated ones. In addition, the $z_{e}$ values predicted for the oil/medium interface are close to those predicted in Refs. [6] and [7] for boundaries without reflection. Our approach is able to explain the overall trend of the scattering anisotropy effects, without adding much complexity to the boundary condition implemented by ZPW.

\section{CONCLUSIONS}

The results presented in this paper show that subtle boundary effects cannot be accounted for by a simple extension of bulk phenomena; a boundary condition only in terms of transport mean free path and reflectivity alone is not sufficient to describe the diffusion phenomenon close to the interface. The extrapolation length ratio $z_{e}$ defines uniquely the relationship between the energy density at the boundary and its gradient normal to the surface. We present evidence that $z_{e}$ depends not only on the reflectivity at the boundary, but also on the anisotropy of the single-scattering process. Therefore, at the interface, the behavior of the energy density is a function of both the diffusive properties of the scattering medium and the particularities of individual scattering centers. We found that $z_{e}$, this additional length scale describing interfacial phenomena, decreases for larger anisotropy factors when the light is detected in a medium of lower refractive index than that of the scattering medium. When the light exits the sample into a medium of higher refractive index, the dependence of $z_{e}$ on $g$ is reversed and, since the reflectivity is lower, it is much weaker.

The present experimental investigation was possible by adapting a recently proposed technique, based on optical path-length resolved measurements. The path-length resolved distribution of backscattered light, which contains the global effect of reflection at the boundary, is used to determine the value of $z_{e}$. Due to the high dynamic range, a broad domain of the optical path length is resolved, which allows a thorough investigation of the diffusive backscattering light. The experimental data clearly illustrate the influence of the boundary properties on the optical path-length distribution of the backscattered light.

A numerical algorithm was designed to simulate the experiment and the results are similar to the experimental ones. In addition, a simple analytical model was introduced to describe the data. In the overall description of the angular distribution of the backscattered waves, our approach emphasizes the particular contribution of the last scattering event. At larger values of $g$, the total angular flux impinging on the physical boundary is more peaked forward and undergoes less reflection than in the case of isotropic scatterers, which corresponds to a smaller value of $z_{e}$.

The results presented here have important implications upon any diffusion experiment that involves bounded scattering media since the knowledge of $z_{e}$ is required for an accurate interpretation of the experimental results. For medical applications, our findings have a special relevance, since the biological tissues are usually characterized by high anisotropy factors.

\section{ACKNOWLEDGMENTS}

This research was partially supported by the National Science Foundation and by the Florida High Tech Corridor Council.
[1] Akira Ishimaru, Wave Propagation and Scattering in Random Media (Academic, New York, 1978).

[2] S. R. Arridge, Inverse Probl. 15, R41-83 (1999).

[3] Advances in Optical Imaging and Photon Migration, OSA
Technical Digest Series Vol. XXI, edited by J. G. Fujimoto and M. S. Patterson (Optical Society of America, Washington, D.C., 1998).

[4] James J. Duderstadt and Louis J. Hamilton, Nuclear Reactor 
Analysis (Wiley, New York, 1976).

[5] M. U. Vera, P.-A. Lemieux, and D. J. Durian, J. Opt. Soc. Am. A 14, 2800 (1997).

[6] E. Amic, J. M. Luck, and Th. M. Nieuwenhuizen, J. Phys. A 29, 4915 (1996).

[7] M. C. W. van Rossum and Th. M. Nieuwenhuizen, Rev. Mod. Phys. 71, 313 (1999).

[8] Nan Guang Chen and Jing Bai, Phys. Rev. Lett. 80, 5321 (1998)

[9] G. Popescu and A. Dogariu, Opt. Lett. 24, 442 (1999).

[10] Richard C. Haskell, J. Opt. Soc. Am. A 11, 2727 (1994).

[11] Ad Lagendijk, Rob Vreeker, and Pedro De Vries, Phys. Lett. A 136, 81 (1989).

[12] Isaac Freund, Phys. Rev. A 45, 8854 (1992).
[13] J. X. Zhu, D. J. Pine, and D. A. Weitz, Phys. Rev. A 44, 3948 (1991).

[14] M. U. Vera and D. J. Durian, Phys. Rev. E 53, 3215 (1996).

[15] D. J. Durian, Phys. Rev. E 50, 857 (1994).

[16] D. J. Durian, Opt. Eng. (Bellingham) 34, 3344 (1995).

[17] S. McMurry, D. Weaire, J. Lunney, and S. Hutzler, Opt. Eng. (Bellingham) 33, 3849 (1994).

[18] S. McMurry, D. Weaire, J. Lunney, and S. Hutzler, Opt. Eng. (Bellingham) 34, 3345 (1995).

[19] L. G. Henyey and J. L. Greenstein, Astrophys. J. 93, 70 (1941).

[20] Michael S. Patterson, B. Chance, and B. C. Wilson, Appl. Opt. 28, 2331 (1989). 